\title{
Radiofrecuencia pulsada (Rf) y ozono intradiscal en el alivio del dolor discogénico: experiencia en 51 casos
}

\author{
L. Cánovas, J. Oduña, A. Huete, L. Alonso, M. Couñago y S. Rojas \\ Servicio de Anestesiología y Reanimación. Unidad de Dolor. Complejo Hospitalario Universitario de \\ Ourense. Ourense
}

Cánovas L, Oduña J, Huete A, Alonso L, Couñago M, Rojas S. Radiofrecuencia pulsada (Rf) y ozono intradiscal en el alivio del dolor discogénico: experiencia en $51 \mathrm{ca-}$ sos. Rev Soc Esp Dolor 2015; 22(1): 27-31.

\begin{abstract}
Objective: About $40 \%$ cases of lumbosacral pain has source in intervertebral disc. Degeneration and nucleus pulposus dehydration can produce tears in the annulus that stimulates growth irrigation and nocioceptors formation at this level and this could be the cause of discogenic pain. Intradiscal radiofrecuency (Rf) has $2 \mathrm{~B} \pm$ evidence level for the discogenic pain relief. Our goal was to test if addition of intradiscal ozone to intradiscal Rf treatment could improve outcomes.

Material and methods: Observational prospective study in which fifty-one patients with discogenic pain were treated. Inclusion criteria: a) Pain's evolution higher than eight months; b) VAS higher than seven; c) non radicular pain characteristics; d) lumbosacral pain with uni- or bilateral buttock irradiation; e) without response to pharmacologic and interventional treatment; f) discal degeneration o presence of a high-intensity zone in RM; g) discography with $30 \%$ ozone: Not evidence of fuga to epidural space with addition of 3-8 ml ozone in nucleus pulposus. Patients were randomized in three groups: A - Just intradiscal ozone (3-8 ml). B - Intradiscal ozone and next RfP (2 Hz, 10 miliseg, $60 \mathrm{~V}$ ) for 15 minutes (Neurotherm). C - Intradiscal - Pulsed Rf (with same parameters). Pulsed Rf parameters were recorded during the procedure: impedance, temperature, voltage and $\mathrm{mA}$. Patients were evaluated at three and twelve months of the technique. Parameters evaluated were:
\end{abstract}

Recibido: 11-09-13.

Aceptado: 15-02-14
EVA, improve percentage (no effect/EVA > 2/EVA > $50 \%$ ). Chosen statistical program was SPSS 16.0, t-student for average comparison, $\mathrm{p}$-simple binomial for percentage comparison $(\mathrm{p}<0.05)$.

Results: More EVA reduction at three and twelve months on the $\mathrm{B}$ group $(8.1 \pm 1.1 / 3.7 \pm 1.9 / 4 \pm 2.2)(\mathrm{p}<0.01)$. All patients from $B$ group significantly improve at three months and $88.2 \%$ of the patients' improvement continuous for twelve months $(\mathrm{p}<0.05)$.

Conclusions: The association of intradiscal ozone with pulsed $\mathrm{Rf}$ can improve the results of the ozone's application in discogenic pain.

Key words: Discal ozone. Pulsed radiofrecuency (RfP). Discogenic pain.

\section{RESUMEN}

Objetivo: aproximadamente el $40 \%$ de los casos de dolor lumbosacro crónico tiene su origen en el disco intervertebral. La degeneración y deshidratación del núcleo pulposo pueden producir desgarros en el anillo fibroso que estimulan el crecimiento de vasos sanguíneos y nociceptores a este nivel que pueden dar lugar al dolor discogénico. La radiofrecuencia (Rf) intradiscal tiene un nivel de evidencia $2 \mathrm{~B} \pm$ en el alivio del dolor discogénico. Nuestro objetivo fue comprobar si la radiofrecuencia pulsada (RfP) intradiscal combinada con ozono intradiscal podría mejorar los resultados.

Material y métodos: estudio prospectivo, observacional de 51 pacientes diagnosticados de dolor discogénico de $>8$ meses de evolución (lumbosacro irradiado a nalga, uni o bilateral, no radicular, sin respuesta a tratamiento farmacológico y/o intervencionista), severo EVA 7-9, con degeneración o zona de alta densidad discal en RM, y discografía con ozono $30 \%$ (3-8 ml) sin fuga al espacio epidural, a través de aguja de Rf $15 \mathrm{~cm}$, $20 \mathrm{~mm}, 20 \mathrm{G}$, con punta emplazada en núcleo pulposo. Los pacientes fueron clasificados aleatoriamente en 3 grupos: A sólo ozono intradiscal; $\mathrm{B}$ - en los que posteriormente al ozo- 
Rev. Soc. Esp. del Dolor, Vol. 22, N. ${ }^{\circ}$ 1, Enero-Febrero 2015

no intradiscal se realizó RfP (2 Hz, 10 miliseg, $60 \mathrm{~V}$ ) durante 15 min (Neurotherm); y C - Rf pulsada intradiscal (con los mismos parámetros). Los parámetros de lesión de Rf pulsada: temperatura, impedancia, voltaje y $\mathrm{mA}$ fueron registrados durante el procedimiento. Los pacientes fueron evaluados a los 3 y 12 meses de la técnica: eficacia analgésica (EVA); \% mejoría (no efecto/EVA > 2/EVA > 50 \%). Estadística con SPSS 16.0. t-Student para comparación de medias. $\mathrm{p}$ binomial simple para comparación de proporciones $(p<0,05)$.

Resultados: mayor reducción del EVA a los 3 y 12 meses en el grupo B $(8,1 \pm 1,1 / 3,7 \pm 1,9 / 4 \pm 2,2)(p<0,01)$. Todos los pacientes del $\mathrm{B}$ mejoraron de forma significativa a los 3 meses $y$ en el 88,2\% esta mejoría continuó a los 12 meses $(p<0,05)$.

Conclusiones: el ozono intradiscal mejoró los resultados de la RfP en el dolor discogénico en este estudio.

Palabras clave: Ozono intradiscal. Radiofrecuencia pulsada (RfP). Dolor discogénico.

\section{INTRODUCCIÓN}

El disco intervertebral desempeña el papel más importante en la estabilización de la columna vertebral. La degeneración discal es un proceso fisiológico con el envejecimiento. La degeneración y deshidratación del núcleo pulposo producen una alteración de la biomecánica del disco que implica a otras estructuras como las placas vertebrales terminales y las articulaciones facetarias, y pueden producir desgarros en el anillo fibroso que estimulan el crecimiento de vasos sanguíneos y nociceptores a este nivel, dando lugar al dolor discogénico (1). Aproximadamente el $40 \%$ de los casos de dolor lumbosacro crónico tiene su origen en el disco intervertebral.

Se han postulado diversas estrategias intervencionistas para el tratamiento del dolor lumbar crónico, de origen discal, que no responde al tratamiento conservador, que incluyen: reducción de inflamación, ablación de nociceptores discales por radiofrecuencia, reducción de presión intradiscal y eliminación de la hernia del núcleo pulposo (2).

$\mathrm{La} \mathrm{Rf}$ intradiscal tiene un nivel de evidencia $2 \mathrm{~B} \pm$ en el alivio del dolor discogénico. No se recomienda la termocoagulación del disco, con Rf, con una sola aguja (2B-). Curiosamente, una técnica poco utilizada, la ablación por Rf del ramo comunicante tiene un nivel de evidencia $2 \mathrm{~B}+$. Actualmente hay estudios suficientes para recomendar la terapia electrotérmica intradiscal (IDET) (2B \pm ) y la biacuplastia (0) (3). Recientemente Rohof (4) publicó buenos resultados con la Rf pulsada intradiscal, con una aguja al año de la técnica.

Otras técnicas como la discólisis con ozono, nucleoplastia y la descompresión discal percutánea pueden utilizarse dentro de un estudio protocolizado. El ozono intradiscal ha sido utilizado con buenos resultados en el tratamiento del dolor discogénico (5-7) por sus efectos demostrados sobre el disco intervertebral y sobre el núcleo pulposo $(8,9)$.
Nuestro objetivo fue comprobar si la Rf pulsada intradiscal combinada con ozono intradiscal podría mejorar los resultados de estas dos técnicas por separado.

\section{MATERIAL Y MÉTODO}

Una vez aprobado el estudio por el Comité de Ética del Centro y obtenido el consentimiento oral y escrito de cada técnica por parte de los pacientes, se realizó un estudio prospectivo, observacional, de 12 meses de evolución de 51 pacientes diagnosticados de dolor discogénico. Los criterios de inclusión fueron:

- Dolor de > 8 meses de evolución (lumbosacro irradiado a nalga, uni o bilateral, no radicular, que aumenta con las maniobras de Valsalva y flexión del tronco, sin respuesta a tratamiento farmacológico y/o intervencionista, incluyendo bloqueo epidural y facetario).

- Severo EVA entre 7-9.

- Con imagen de degeneración discal, pérdida de altura del disco, o zona de alta densidad en la RM en T2 que sugiere rotura del anillo fibroso a ese nivel.

- Discografía con ozono $30 \%$ (3-8 ml) sin fuga al espacio epidural (Fig. 1), mediante de aguja de Rf $15 \mathrm{~cm}$, $20 \mathrm{~mm}, 20 \mathrm{G}$, con punta emplazada en núcleo pulposo del nivel a tratar según RM.

Los pacientes fueron clasificados aleatoriamente en 3 grupos $(\mathrm{n}=17)$ mediante un programa informático. El tratamiento se llevó a cabo, en todos los casos, bajo control de escopia:

- A ( $\mathrm{n}=17)$ : sólo ozono intradiscal.

- B ( $n=17)$ en los que posteriormente al ozono intradiscal se realizó Rf pulsada intradiscal, con punta de aguja en núcleo pulposo $(2 \mathrm{~Hz}, 10$ miliseg, $60 \mathrm{~V})$ durante 15 min (Neurotherm), protocolo utilizado por Rohof (4).

- C ( $\mathrm{n}=17)$ : Rf pulsada intradiscal con los mismos parámetros utilizados en el grupo B (Figs. 1-3).

Los parámetros de lesión de Rf pulsada: temperatura, impedancia, voltaje y $\mathrm{mA}$ fueron registrados durante el procedimiento. Todo el procedimiento fue realizado bajo sedación con $50 \mu \mathrm{g}$ de fentanilo $+2 \mathrm{mg}$ de midazolam. Se utilizó profilaxis antibiótica con $200 \mathrm{mg}$ de ciprofloxacino intravenoso.

Los pacientes fueron evaluados a los 3 y 12 meses de la técnica. Las variables estudiadas fueron:

- Disco lumbar a abordar.

- Eficacia analgésica (EVA).

- \% mejoría, considerando:

- No efecto.

- Descenso en el EVA $>2$.

- Reducción del EVA $>50 \%$ respecto a la visita basal.

- Efectos indeseables.

- Impresión global de cambio por parte del paciente (1-7; 1: muchísimo mejor y 7: muchísimo peor), a los 12 meses. 


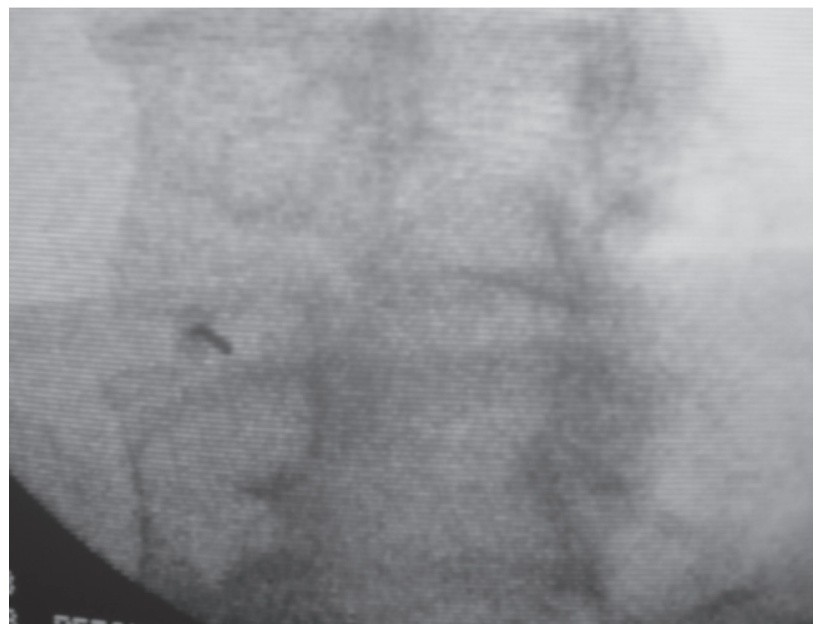

Fig. 1. Abordaje del disco por escopia en anteroposterior.

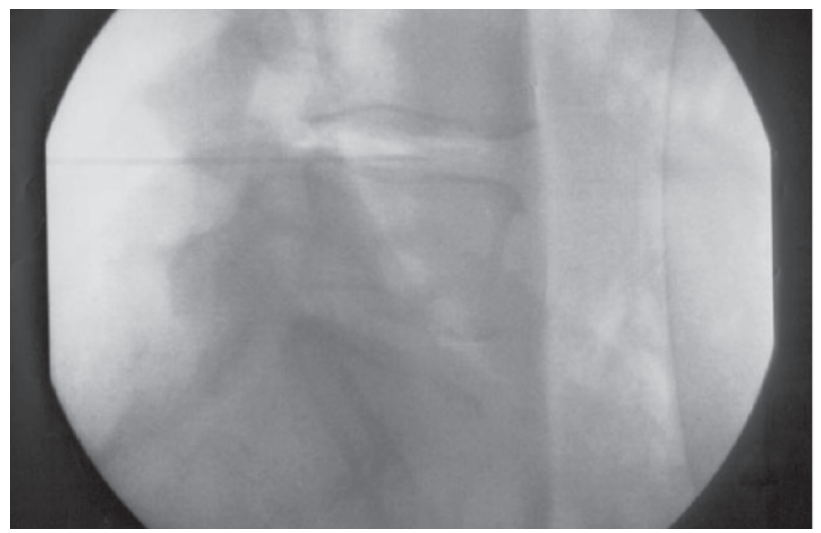

Fig. 2. Ozono intradiscal.

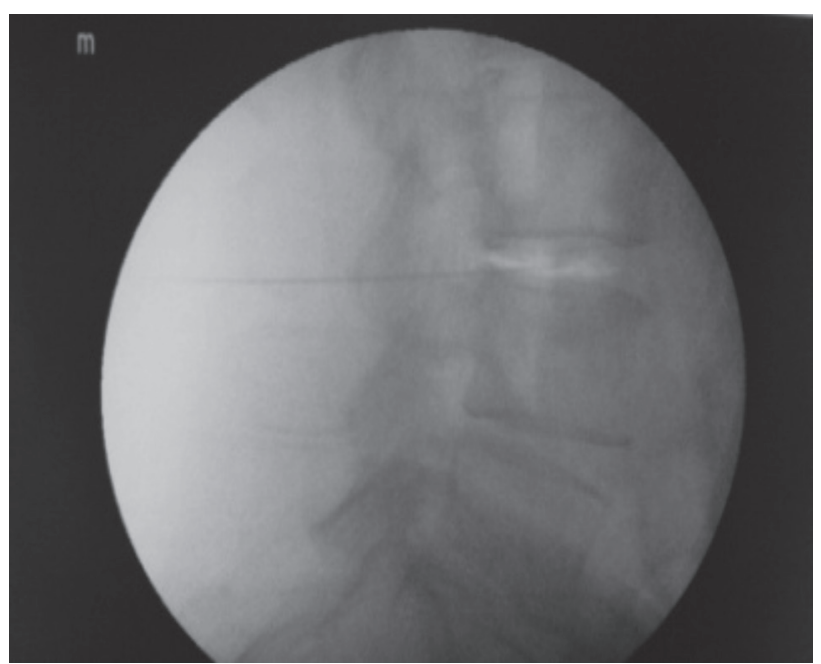

Fig. 2. Rf pulsada y ozono intradiscal.
El estudio estadístico se realizó con el programa SPSS 16.0. Se utilizó la t-Student para la comparación de medias y prueba binomial simple para comparación de proporciones. Se consideró significativo una $\mathrm{p}<0,05$.

\section{RESULTADOS}

Los niveles discales tratados se representan en la tabla I.

La reducción del EVA a los 3 y 12 meses fue significativa en los tres grupos respecto a la visita basal. Los valores del EVA en el grupo en el que se realizó Rf pulsada + ozono fue inferior a los otros dos grupos, tanto en la revisión de los tres meses como en la de 12 meses. A los tres meses las diferencias no fueron significativas, pero a los 12 meses se encontraron diferencias significativas con respecto al EVA del grupo ozono $(\mathrm{p}=0,02)$ y con respecto al EVA del grupo Rf pulsada ( $\mathrm{p}=0,009)$ (Tabla II).

Todos los pacientes del grupo Rf pulsada + ozono mejoraron en la revisión de los tres meses. El 70,5\% de dichos pacientes obtuvieron en esta revisión una mejoría $>50 \%$ $(\mathrm{p}<0,000)$.

En la revisión de los 12 meses, el número de pacientes que mantuvieron una mejoría $>50 \%$ en el EVA fue superior en el grupo $\mathrm{Rf}+$ ozono respecto al grupo ozono $(\mathrm{p}<$ $0,05)$ y respecto al grupo $\mathrm{Rf}(\mathrm{p}<0,00)$. El 88,2 \% de los pacientes del grupo $\mathrm{Rf}+$ ozono, mantuvieron algún tipo de mejoría a los 12 meses de la técnica (Tabla III).

No se registraron complicaciones de sangrado epidural, discitis u otras infecciones relacionadas con el procedimiento. Tampoco cefaleas ni déficit motor o sensorial.

Con respecto a la impresión global de cambio según el paciente, todos los pacientes del estudio estaban con una puntuación entre 1 (muchísimo mejor) y 4 (ningún cambio). En el grupo A, el 65,6 \% de los pacientes refirieron puntuaciones 1 y 2 (muchísimo mejor/mucho mejor), frente al 88,2 \% del grupo B y 70,5\% del grupo C (p<0,01).

\section{DISCUSIÓN}

En nuestro estudio, la asociación de Rf pulsada y ozono intradiscal, mejoró la eficacia analgésica a los 3 y 12 meses

TABLA I. NIVEL DISCAL TRATADO EN CADA GRUPO

\begin{tabular}{lccc}
\hline $\begin{array}{c}\text { Discos } \\
\text { tratados }\end{array}$ & Ozono $(A)$ & $\begin{array}{c}\text { Rf pulsada } \\
\text { + ozono }(B)\end{array}$ & $\begin{array}{c}\text { Rf pulsada } \\
(C)\end{array}$ \\
\hline L2-L3 & 1 & 2 & 2 \\
L4-L5 & 8 & 7 & 8 \\
L5-S1 & 8 & 8 & 7 \\
\hline
\end{tabular}


TABLA II. EFICACIA ANALGÉSICA MEDIDA POR EL EVA A LOS 3 Y 12 MESES DE LA TÉCNICA

\begin{tabular}{llll}
\hline \multicolumn{1}{c}{ EVA } & \multicolumn{1}{c}{ Ozono $(A)$} & \multicolumn{1}{c}{ Rfpulsada + ozono $(B)$} & \multicolumn{1}{c}{ Rf pulsada $(C)$} \\
\hline Basal & $7,8 \pm 1,3$ & $8,1 \pm 1,1$ & $7,9 \pm 2,1$ \\
3 meses & $4,2 \pm 1,7(p<0,0001)$ & $3,7 \pm 1,9(p<0,0001)$ & $3,9 \pm 2,3(p=0,0007)$ \\
12 meses & $5,9 \pm 1,1(p=0,0024)$ & $4 \pm 2,2(p<0,0001)$ & $6,2 \pm 0,9(p=0,03)$ \\
\hline
\end{tabular}

Valores expresados en $\mathrm{x} \pm \mathrm{ds}$.

TABLA III. PORCENTAJE DE MEJORÍA DE LOS PACIENTES A LOS 3 Y 12 MESES DE LA TÉCNICA

\begin{tabular}{lllll}
\hline Eficacia analgésica & \multicolumn{1}{c}{$\begin{array}{c}\text { Ozono }(A) \\
(n=17) \\
n(\%)\end{array}$} & $\begin{array}{c}\text { Rfpulsada+ozono } \\
(B) \\
(n=17) \\
n(\%)\end{array}$ & $\begin{array}{c}\text { Rf pulsada }(C) \\
(n=17) \\
n(\%)\end{array}$ \\
\hline \multirow{3}{*}{3 meses } & Nula & $3(17,6)$ & $0(0)$ & $5(29,4)$ \\
& Mejoría EVA $>2$ & $6(35,2)$ & $5(29,4)$ & $6(35,2)$ \\
& Mejoría EVA $>50 \%$ & $8(47,05)$ & $12(70,5)(p<0,000)$ & $6(35,2)$ \\
& Nula meses & $5(29,4)$ & $2(11,76)$ & $4(23,5)$ \\
& Mejoría EVA $>2$ & $5(29,4)$ & $5(29,4)$ & $7(41,1)$ \\
& Mejoría EVA $>50 \%$ & $7(41,1)(p<0,05)$ & $10(58,8)$ & $6(35,2)(p<0,00)$ \\
\hline
\end{tabular}

Valores expresados en $\mathrm{n}$ y $\%$.

de realizada la técnica, con respecto a ambas técnicas por separado.

El diagnóstico de dolor discogénico se llevó a cabo por la clínica, intensidad y maniobras de provocación del dolor, unido a imagen discal de alta densidad en la RM, que presenta una correlación del $80 \%$ con la presencia de dolor discogénico y de citoquinas inflamatorias a ese nivel (10). La falta de respuesta a otras técnicas como la infiltración facetaria o epidural nos permitió también excluir otros posibles diagnósticos.

Existen dos tipos de cambios de químicos que ocurren en el disco degenerado. En primer lugar, una fractura en la placa de extremo vertebral puede conducir a la introducción de citoquinas inflamatorias en el núcleo pulposo. Esta respuesta inflamatoria cambia el delicado equilibrio de nutrientes en el núcleo, resultando en una disminución de la oxigenación, incremento en la concentración local de lactato y disminución en el pH del disco. En algunos casos, las citoquinas inflamatorias pueden ser, por sí mismas, la causa del dolor, y la ruptura anular externa puede facilitar la "fuga" de estos mediadores inflamatorios a estructuras adyacentes epidurales como el ligamento longitudinal posterior, la dura y el ganglio de raíz dorsal. El crecimiento de nociceptores en las capas profundas del disco puede sensibilizar el disco a las cargas mecánicas normales. Estos mecanismos pueden causar una sensibilización "química o mecánica" del disco (11).

El ozono intradiscal podría disminuir el volumen del disco por un mecanismo que incluye la salida de moléculas de agua y la degeneración celular de la matriz por medio de la oxidación de proteoglicanos (12). Esto originaría una pérdida de volumen, una menor compresión venosa, y un menor éstasis venoso, mejorando la microcirculación local y aumentando el aporte de oxígeno a la raíz nerviosa, lo cual disminuye el dolor neuropático, fenómeno demostrado en estudios animales (13). Otros estudios justificaron el efecto analgésico y antiinflamatorio del ozono por la inhibición de la síntesis de prostaglandinas y bradicinina, la estimulación de citoquinas inmunosupresoras (IL10) o factor transformador del crecimiento beta 1 , y la inhibición de la síntesis de citoquinas inflamatorias como IL1, IL2, IL8, IL12, IL15, interferón alfa o factor de necrosis tumoral alfa (14).

Varios estudios han demostrado la eficacia del ozono en el alivio del dolor discogénico $(6,7,15)$.

Se ha publicado que las técnicas de anuloplastia por radiofrecuencia aplicada al núcleo pulposo podrían alterar la conductividad de las terminaciones nerviosas, lo que ocasionaría una reducción del dolor, y también podrían activar el sistema inmune, reduciendo de este modo el proceso inflamatorio del dolor crónico (4). El campo eléctrico, generado en el núcleo pulposo, modularía la respuesta inmune tanto a nivel del sistema nervioso central como periférico. La Rf pulsada modula localmente la expresión de citoquinas por una inhibición de las células de Schwann al mismo tiempo que se produce una activación de astrocitos a nivel de la médula espinal. Estos cambios están asociados a cambios en la modulación del dolor (16). 
La Rf pulsada intradiscal se ha mostrado eficaz en el tratamiento del dolor discogénico en varios estudios. Sin embargo los resultados en cuanto a pacientes que mantienen alivio del dolor mayor al $50 \%$ a los 3 y 12 meses de la técnica, son inferiores a los obtenidos en nuestro grupo Rf pulsada + ozono $(4,17,18)$.

Gautam y cols. (19), de forma similar, obtienen una mejoría del dolor mayor con la utilización de ozono asociado a termocoagulación del disco que con ozono sólo, tanto a los 3 como a los 12 meses de la técnica. En nuestro estudio la asociación de Rf pulsada intradiscal al ozono supera en eficacia analgésica a la publicada en el estudio de Gautam y cols. (19), a lo que hay que añadir que la evidencia disponible no apoya la eficacia o efectividad de la termocoagulación intradiscal en el tratamiento del dolor discogénico (20).

A pesar de la limitación del número de casos, la asociación de Rf intradiscal y ozono ha mejorado los resultados y esta mejoría ha sido percibida de forma positiva por los pacientes, sin embargo habría sido interesante el haber incluido test de calidad de vida y/o funcionalidad. Son necesarios estudios futuros que incluyan un mayor número de pacientes, más estrictos criterios de inclusión y evaluaciones a más largo plazo, no sólo de eficacia analgesia sino también de calidad de vida.

\section{CORRESPONDENCIA:}

Luz Cánovas

Servicio de Anestesiología y Reanimación

Unidad de Dolor

Complejo Hospitalario Universitario de Ourense

C/ Ramón Puga Noguerol, 54

32005 Ourense

e-mail: maria.de.la.luz.canovas.martinez@sergas.es

\section{BIBLIOGRAFÍA}

1. Roh JS, Teng AL, Yoo JU, Davis J, Furey C, Bohlman HH. Degenerative disorders of the lumbar and cervical spine. Othop Clin N Am 2005;36:255-62.

2. Kallewaard JW, Terheggen MAMB, Groen GJ, Sluijter ME, Derby R, Kapural L, et al. Discogenic Low Back Pain. In: van Zundert J, Patijn J, Hartrick CT, Lataster A, Huygen FJPM, Mekhail N, van Kleef M, editors. Evidence-Based Interventional Pain Medicine: According to Clinical Diagnoses. Oxford, UK: Wiley-Blackwell; 2011.

3. Van Zundert J, Hartrick CT, Patijn J, Huygen F, Mekhail $\mathrm{N}$, van Kleef M. Evidence based interventional pain medicine according to clinical diagnoses. Pain Practice 2011; 11:423-9.
4. Rohof O. Intradiscal pulsed radiofrequency application following provocative discography for the management of degenerative disc disease and concordant pain: A pilot study. Pain Practice 2012;12:342-9.

5. Gallucci M, Limbucci N, Zugaro L, Barile A, Stavroulis E, Ricci A, et al. Sciatica: Treatment with intradiscal and intraforaminal injections of steroid and oxygen-ozone versus steroid only. Radiology 2007;242:907-13.

6. Wang P, Chen C, Liu B. CT guided oxygen-ozone injection for the treatment of discogenic back pain. China Practical Medicine 2012;7:61-8.

7. Zhao B, Shao GH, Yu Y, Zhou YF, Zhong B, He C. Preliminary report for treatment of discogenic low back pain with combined percutaneous laser and O2-O3 mixture. Zhongguo Gu Shang 2008;21:391-2.

8. Zhou CW, Deng C, Zhou HC, Liu JF. Study of the related factors with immediate effect of oxygen-ozone on intervertebral disc nucleus pulposus tissue. Chinese Journal of Pain Medicine 2011;5.

9. Re L, Sanchez GM, Mawsouf N. Clinical evidence of ozone interaction with pain mediators. Saudi Med J 2010; 31:1363-77.

10. Wolfer LR, Derby R, Lee JE, Lee SH. Systematic review of lumbar provocation discography in asymptomatic subjects with a meta-analysis of false-positive rates. Pain Physician 2008;11:513-38.

11. Burke JG, Watson RW, McCormack D, Dowling FE, Walsh MG, Fitzpatrick JM. Intervertebral discs which cause low back pain secrete high levels of proinflammatory mediators. J Bone Joint Surg Br 2002;84:196-201.

12. Johnson BA. Therapeutic periradicular injections: It's a gas! AJNR Am J Neuroradiol 2005;26:988-9.

13. Steppan J, Meaders T, Muto M, Murphy K. A metaanalysis of the effectiveness and safety of ozone treatments for herniated lumbar discs. Vasc Interv Radiol 2010;21:534-48.

14. Iliakis E, Valadakis V, Vynios DH, Tisiganos CP, Agapitos E. Rationalization of the activity of medical ozone on intervertebral disc: A histological and biochemical study. Riv Neuroradiol 2001;14:23-30.

15. Das G, Ray S, Iswarari S, Roy M, Ghosh P. Ozone nucleolysis for management of pain and disability in prolapsed lumber intervertebral disc: A prospective cohort study. Interventional Neuroradiology 2009;15:330-4.

16. Vallejo R, Tilley D, Vogel L, Benyamin R. Pulsed radiofrequency modulates cytokine expression in the spared nerve injury model. Reg Anesth Pain Med 2009;2:24.

17. Fukui S, Nitta K, Iwashita N, Tomie H, Nosaka S, Rohof O. Intradiscal pulsed radiofrequency for chronic lumbar discogenic low back pain: A one year prospective outcome study using discoblock for diagnosis. Pain Physician 2013;16:435-42.

18. Teixeira A, Sluijter ME. Intradiscal highvoltage, long-duration pulsed radiofrequency for discogenic pain: A preliminary report. Pain Med 2006;7:424-8.

19. Gautam S, Rastogi V, Jain A, Singh AP. comparative evaluation of oxygen-ozone therapy and combined use of oxygen-ozone therapy with percutaneous intradiscal radiofrequency thermocoagulation for the treatment of lumbar disc herniation. Pain Practice 2010;11:160-6.

20. Urrutia G, Kovacs F, Nishishinya MB, Olabe J. Percutaneous thermocoagulation intradiscal techniques for discogenic low back pain. Spine 2007;32:1146-54. 